\title{
Crystal structure of the zinc-, cobalt-, and iron-containing adenylate kinase from Desulfovibrio gigas: a novel metal-containing adenylate kinase from Gram-negative bacteria
}

\author{
A. Mukhopadhyay $\cdot$ A. V. Kladova $\cdot$ S. A. Bursakov $\cdot$ O. Yu. Gavel $\cdot$ J. J. Calvete \\ V. L. Shnyrov $\cdot$ I. Moura $\cdot$ J. J. G. Moura $\cdot$ M. J. Romão $\cdot$ J. Trincão
}

Received: 21 April 2010/Accepted: 2 August 2010/Published online: 7 September 2010

(c) SBIC 2010

\begin{abstract}
Adenylate kinases (AK) from Gram-negative bacteria are generally devoid of metal ions in their LID domain. However, three metal ions, zinc, cobalt, and iron, have been found in AK from Gram-negative bacteria. Crystal structures of substrate-free AK from Desulfovibrio
\end{abstract}

An Interactive 3D Complement page in Proteopedia is available at: http://proteopedia.org/wiki/index.php/Journal:JBIC:1.

Electronic supplementary material The online version of this article (doi:10.1007/s00775-010-0700-8) contains supplementary material, which is available to authorized users.

A. Mukhopadhyay · A. V. Kladova · S. A. Bursakov ·

O. Yu. Gavel · I. Moura · J. J. G. Moura .

M. J. Romão $(\bowtie) \cdot$ J. Trincão $(\square)$

REQUIMTE, Departamento de Química,

Centro de Química Fina e Biotecnologia,

Faculdade de Ciências e Tecnologia,

Universidade Nova de Lisboa,

2829-516 Caparica, Portugal

e-mail: mromao@dq.fct.unl.pt

J. Trincão

e-mail: trincao@dq.fct.unl.pt

\section{S. A. Bursakov}

Departamento de Protección Ambiental,

Estación Experimental del Zaidin (EEZ-CSIC),

18008 Granada, Spain

O. Yu. Gavel · V. L. Shnyrov

Departamento de Bioquímica y Biología Molecular,

Facultad de Biología,

Universidad de Salamanca,

37007 Salamanca, Spain

J. J. Calvete

Instituto de Biomedicina de Valencia, CSIC,

Jaume Roig 11, 46010 Valencia, Spain gigas with three different metal ions $\left(\mathrm{Zn}^{2+}, \mathrm{Zn}-\mathrm{AK} ; \mathrm{Co}^{2+}\right.$, $\mathrm{Co}-\mathrm{AK}$; and $\left.\mathrm{Fe}^{2+}, \mathrm{Fe}-\mathrm{AK}\right)$ bound in its LID domain have been determined by X-ray crystallography to resolutions 1.8, 2.0, and 3.0 А, respectively. The zinc and iron forms of the enzyme were crystallized in space group I222, whereas the cobalt-form crystals were $C 2$. The presence of the metals was confirmed by calculation of anomalous difference maps and by X-ray fluorescence scans. The work presented here is the first report of a structure of a metal-containing AK from a Gram-negative bacterium. The native enzyme was crystallized, and only zinc was detected in the LID domain. Co-AK and Fe-AK were obtained by overexpressing the protein in Escherichia coli. $\mathrm{Zn}-\mathrm{AK}$ and $\mathrm{Fe}-\mathrm{AK}$ crystallized as monomers in the asymmetric unit, whereas Co-AK crystallized as a dimer. Nevertheless, all three crystal structures are very similar to each other, with the same LID domain topology, the only change being the presence of the different metal atoms. In the absence of any substrate, the LID domain of all holoforms of $\mathrm{AK}$ was present in a fully open conformational state. Normal mode analysis was performed to predict fluctuations of the LID domain along the catalytic pathway.

Keywords Adenylate kinase - Gram-negative bacteria . Zinc $\cdot$ Cobalt $\cdot$ Iron
Abbreviations
AK Adenylate kinase
AK2 Adenylate kinase isoform 2
Co-AK $\mathrm{Co}^{2+}$ form of adenylate kinase
Fe-AK $\mathrm{Fe}^{2+}$ form of adenylate kinase
NMA Normal mode analysis
PDB Protein Data Bank
$\mathrm{Zn}$-AK $\mathrm{Zn}^{2+}$ form of adenylate kinase 


\section{Introduction}

Adenylate kinase (ATP:AMP phosphotransferase, EC 2.7.4.3; AK) is an essential catalyst for cellular growth and multiplication. AK belongs to a family of enzymes essential to life [1], and is highly abundant inside the cell. It is involved in the reversible transfer of the terminal phosphate group from $\mathrm{Mg}^{2+} \mathrm{ATP}$ to $\mathrm{Mg}^{2+} \mathrm{AMP}$ with high energy turnover: $\mathrm{Mg}^{2+} \mathrm{ATP}+\mathrm{AMP} \leftrightarrow \mathrm{Mg}^{2+} \mathrm{ADP}+$ ADP [2].

AK are $\alpha / \beta$ proteins (a five-stranded $\beta$-sheet surrounded by several $\alpha$-helices) [3-5]. They are composed of three main domains: LID, core and AMP binding. The LID and AMP binding regions undergo significant conformational changes during the catalytic reaction, generating a closed cavity where the reaction takes place, isolated from the bulk solvent [6]. The temperature dependence of the catalytic activity is controlled by these two mobile domains [7]. There are two oligomeric classes of AK: monomeric, as found in Eubacteria, and trimeric, found in Archaebacteria [8]. The sequence and structure identity of both classes are related only distantly, suggesting that archaeal AK belong to a different class [9]. AK from Paracoccus denitrificans was reported to be dimeric [10], but no structural data are available. Simultaneous coexistence of monomeric and dimeric forms and supramolecular assembly of AK was also observed [11].

$\mathrm{AK}$ are divided into two groups: the short and the long forms, which differ in a 20-30 amino acid residue insertion in the LID domain [3, 4]. Mammalian AK (both cytosolic and nuclear) are usually of the short form, whereas the long $\mathrm{AK}$ are found in most bacteria, yeast, and mitochondria. In short AK, the LID domain is reduced to a small irregular loop of less than 11 amino acids. However, the rest of the primary structure, the overall fold, and the side chains involved in nucleotide binding and catalysis are conserved among the short and long variants.

The structural metal binding motif in Gram-positive bacteria is Cys- $\mathrm{X}_{2}-\mathrm{Cys}-\mathrm{X}_{16}-\mathrm{Cys}-\mathrm{X}_{2}-\mathrm{Cys} / \mathrm{Asp}$ [12]. The sulfur positions of the four Cys side chains of Gram-positive bacteria are geometrically optimized to bind the metal ion [13]. In AK from Gram-negative bacteria, the four Cys residues are substituted by four highly conserved amino acids, His, Ser, Asp, and Thr, respectively, suggesting that these residues might play a similar structural role [14]. The crystal structure of AK from Escherichia coli shows that the LID domain forms a single distorted antiparallel $\beta$-sheet, two turns, and one loop [15]. The aforementioned four amino acids lie on the distorted $\beta$-sheet, and are involved in the formation of a hydrogen-bond network that stabilizes the LID structure. In AK from Gram-positive bacteria, zinc seems to stabilize the LID domain more readily than does the hydrogen-bond network present in Gram-negative bacteria and in all of the eukaryotic longform AK determined so far [4, 13]. Several studies have shown that the presence of a metal ion in the LID domain causes considerable differences in the overall stability of $\mathrm{AK}$ and its removal leads to partial or complete loss of catalytic activity [7, 12, 14, 16-18].

Previous studies have shown that zinc depletion reduces the temperature at the midpoint of the thermally induced transition $\left(T_{\mathrm{m}}\right)$ by 7.5 and $6.2{ }^{\circ} \mathrm{C}$ compared with that of the holoforms of AK from the thermophilic bacteria Bacillus stearothermophilus and Thermotoga neapolitana, respectively [17, 19], identifying $\mathrm{Zn}^{2+}$ as a stabilizing feature in AK. The removal of metal ions does not affect the phosphorylating activity of apoproteins from Paracoccus denitrificans, E. coli (quadruple Cys mutant $\mathrm{AK}_{\mathrm{e}} \mathrm{C}_{4}$ ), B. stearothermophilus, and T. neapolitana [10, 14, 16, 17].

The following exceptions have been reported for metalcontaining AK from Gram-negative bacteria: AK from Desulfovibrio gigas and Desulfovibrio desulfuricans (with the metal-chelating motif Cys ${ }^{129}-\mathrm{X}_{5}$-His- $\left.\mathrm{X}_{15}-\mathrm{Cys}_{-}-\mathrm{X}_{2}-\mathrm{Cys}\right)$ [19], which contain either cobalt or zinc, AK from $P$. denitrificans (Cys $\left.{ }^{126}-\mathrm{X}_{2}-\mathrm{Cys}-\mathrm{X}_{16}-\mathrm{Cys}-\mathrm{X}_{2}-\mathrm{Cys}\right)$ overproduced in E. coli, which binds either zinc or iron [17], and $\mathrm{AK}$ from T. neapolitana $\left(\mathrm{Cys}^{134}-\mathrm{X}_{2}-\mathrm{Cys}-\mathrm{X}_{16}-\mathrm{Cys}-\mathrm{X}_{2}-\right.$ Cys) and Chlamydia pneumoniae (Cys ${ }^{133}-\mathrm{X}_{2}-\mathrm{Cys}_{-} \mathrm{X}_{12^{-}}$ Cys- $X_{2}$-Cys), which contain zinc $[17,20]$. Thus, up to now, three metal ions, cobalt, zinc, and iron, have been observed in AK from Gram-negative bacteria.

To investigate the role of these three metal ions, and their influence on activity and structural stability of AK from Gram-negative bacteria, AK from D. gigas was overproduced in $E$. coli and homogeneous $\mathrm{Co}^{2+}, \mathrm{Zn}^{2+}$, and $\mathrm{Fe}^{2+}$ forms of $\mathrm{AK}$ (Co-AK, $\mathrm{Zn}-\mathrm{AK}$, and $\mathrm{Fe}-\mathrm{AK}$, respectively) were obtained (metal-to-protein ratio approximately 1:1) [21]. This previous study showed that both the catalytic activity and the thermal stability of the enzyme are affected by the nature of the metal ion present in the LID domain. Both Co-AK and $\mathrm{Zn}-\mathrm{AK}$ show similar activities and thermal stability (with reversible thermal denaturation occurring at 43.7 and $45.3{ }^{\circ} \mathrm{C}$, respectively), whereas $\mathrm{Fe}-\mathrm{AK}$ denatures irreversibly with a $T_{\mathrm{m}}$ of $45.0{ }^{\circ} \mathrm{C}$ [21].

Herein, we report the crystal structures of substrate-free Co-AK, Zn-AK, and Fe-AK at 2.0-, 1.8-, and 3.0- $\AA$ resolution, respectively. So far, the only metal-containing AK structures known are from Gram-positive bacteria. This is the first report of the crystal structure of a metal-containing AK from Gram-negative bacteria. The structural data described here allow the comparison with the other (apo and holo forms) structures reported. 


\section{Materials and methods}

Sample preparation and crystallization

Protein cloning, bacterial growth, and homogeneous protein production with a unique metal ion were reported in our earlier work [21, 22].

D. gigas cells were grown in a $400 \mathrm{~L}$ fermenter under anaerobic conditions in a basal medium using a lactate/ sulfate medium. Cells were harvested at the beginning of the stationary phase and disrupted. The extract was used after centrifugation for the purification of AK. The native $\mathrm{Co}^{2+} / \mathrm{Zn}^{2+}$ from D. gigas contains $0.4 \pm 0.02 \mathrm{~mol}$ cobalt and $0.3 \pm 0.03 \mathrm{~mol}$ zinc per mole of protein [12].

The gene coding for the AK of D. gigas (EMBL Nucleotide Sequence Database accession number FN424087) was cloned and the resulting plasmid pET$22 \mathrm{~b}(+) / \mathrm{AK}$ with the gene inserted was used for protein expression in E. coli strain BL-21(DE3). Translation of the insert gene was induced in mid-log phase $\left(A_{660 \mathrm{~nm}} \approx\right.$ $0.6-0.7)$ by the addition of $1 \mathrm{mM}$ isopropyl 1 -thio- $\beta$-Dgalactopyranoside to minimal M63B1 medium supplemented with $0.4 \%$ glucose at $37{ }^{\circ} \mathrm{C}$ with vigorous shaking. The incubation temperature and the metal concentration in the medium were optimized to obtain homogeneous protein with a single metal. Typically, cells were grown in $1 \mathrm{~L}$ of M63B1 medium containing $100 \mu \mathrm{g} / \mathrm{ml}$ ampicillin and the expression conditions were as follows: for Co-AK, $6 \mathrm{~h}$ and minimal medium supplemented with $160 \mu \mathrm{M} \mathrm{CoCl}_{2}$; for $\mathrm{Zn}-\mathrm{AK}, 4 \mathrm{~h}$ and $250 \mu \mathrm{M} \mathrm{ZnCl}{ }_{2}$; for $\mathrm{Fe}-\mathrm{AK}, 4 \mathrm{~h}$ and $130 \mu \mathrm{M} \mathrm{FeCl}_{2}$ at $37{ }^{\circ} \mathrm{C}$. After these incubation periods, cells were harvested by centrifugation and disrupted. The soluble fraction of the centrifuged extract was then used for the purification of AK.

Purification of the native $\mathrm{AK}$ and the recombinant $\mathrm{AK}$ from E. coli was performed in a two-step process involving fast protein liquid chromatography (Pharmacia) on a Blue Sepharose Fast Flow column followed by gel filtration on a Superdex 75 column at $4{ }^{\circ} \mathrm{C}$.

The quantification of the metals in the pure proteins was performed by inductively coupled plasma atomic emission spectroscopy analysis with a Horiba Jobin-Yvon apparatus (model Ultima). Standard solutions containing cobalt, iron, and zinc were supplied by Aldrich.

Crystals were grown at $277 \mathrm{~K}$ using the hanging drop vapor diffusion method. The best crystallization conditions were $0.2 \mathrm{M}$ sodium/potassium tartrate, $0.1 \mathrm{M} 2$ - $(N$-morpholino)ethanesulfonic acid ( $\mathrm{pH} 6.5$ ), and 20\% PEG 200 or PEG 800 (the protein to well solution ratio in the drop was $1: 1,1: 2$, or $1: 3$, with the final drop volume of 4,6 , or $8 \mu \mathrm{l}$ ) using a protein stock concentration of approximately $10 \mathrm{mg} / \mathrm{ml}$ [22]. The crystals were cryoprotected by soaking them at $277 \mathrm{~K}$ in mother liquor containing $15-30 \%$ glycerol. The crystals were then flash-cooled in a stream of nitrogen gas at $100 \mathrm{~K}$.

Fluorescence scan, diffraction data, structure analysis, and refinement

Diffraction data were collected at beam lines ID-14-4 and ID-29 at the European Synchroton Research Foundation (Grenoble, France) using either a Quantum 4R or a 315R $\mathrm{CCD}$ detector (Area Detector Systems Corporation). All the data sets were processed and scaled using MOSFLM and SCALA from the CCP4 suite [23-25]. Details of data collections and reprocessing of the previously published data are presented in Table 1 [22].

Owing to difficulties in obtaining a molecular replacement solution using models of AK from other organisms, the structure of $\mathrm{Zn}-\mathrm{AK}$ was solved by multiple anomalous dispersion using the bound zinc as the anomalous scatterer. A single crystal was used to collect data to $2.10 \AA$ at the zinc absorption peak $(\lambda=1.2852)$ and at the edge $(\lambda=1.2855)$ and to $1.78 \AA$ at a remote wavelength $(\lambda=1.2825)$. The crystal belongs to space group I222. The structure was solved using AutoSHARP [26] [Protein Data Bank (PDB) accession code 2XB4].

Fluorescence scans were performed with the crystals of $\mathrm{Co}-\mathrm{AK}$ and $\mathrm{Fe}-\mathrm{AK}$ to confirm the presence and type of metal ion in the crystals (Fig. S1). A single data set was collected for Co-AK at the edge $(\lambda=1.6064 \AA)$ with a $\varphi$ rotation of $1^{\circ}$ per image and was used for structure refinement. The structure was solved by molecular replacement with the program PHASER [27] using Zn-AK as a search model (PDB accession code 3L0S)]. Data in the resolution range 44.45-2.00 $\AA$ were used for molecular replacement and refinement. The best solution was obtained in the $C 2$ space group with two molecules in the asymmetric unit. First restrained refinement, after density modification, with 44.45-2.00- $\AA$ data using REFMAC [28] resulted in $R=0.313$ and $R_{\text {free }}=0.320$. For Fe-AK, 180 images were collected, with $\varphi$ rotation of $0.6^{\circ}$ per image, at the iron edge $(\lambda=1.7266 \AA)$. The structure was also solved by molecular replacement using PHASER [27] (PDB accession code 3LOP) using data in the resolution range 59.58-3.00 $\AA$. The best solution was in the $I 222$ space group with a single molecule in the asymmetric unit. First restrained refinement, after the density modification, with 59.58-3.00- $\AA$ data using REFMAC yielded $R=0.307$ and $R_{\text {free }}=0.310$. Iterative model building with COOT [29], guided by $2 m F_{0}-D F_{c}$ and $m F_{0}-D F_{c}$ maps, together with restrained refinement in REFMAC [28], initially, and then with restrained refinement including TLS, resulted in good final models for all the structures (as judged by the validation tools in COOT). The final model of Co-AK includes amino acid residues 1-223, 300 water 
Table 1 Data collection and statistics

\begin{tabular}{|c|c|c|c|c|c|}
\hline \multirow[t]{2}{*}{ Dataset } & \multicolumn{3}{|l|}{ Zn-AK } & \multirow[t]{2}{*}{ Co-AK } & \multirow[t]{2}{*}{$\mathrm{Fe}-\mathrm{AK}$} \\
\hline & Peak & Edge & Remote & & \\
\hline $\mathrm{X}$-ray source & ID-14-4 & & & ID-29 & ID-29 \\
\hline \multicolumn{6}{|l|}{ Crystal data } \\
\hline \multirow[t]{4}{*}{ Unit cell parameters $(\AA)(\mathrm{deg})$} & $a=39.390$ & & & $a=131.530$ & $a=38.800$ \\
\hline & $b=119.440$ & & & $b=39.510$ & $b=119.160$ \\
\hline & $c=149.590$ & & & $c=94.300$ & $c=146.710$ \\
\hline & & & & $\beta=109.430$ & \\
\hline Molecules per ASU & 1 & & & 2 & 1 \\
\hline Mosaicity & 0.73 & & & 0.63 & 0.73 \\
\hline Matthews coefficient $\left(\AA^{3} / \mathrm{Da}\right)$ & 3.59 & & & 2.31 & 3.46 \\
\hline Solvent content $(\%)$ & 65.5 & & & 48.3 & 64.3 \\
\hline Maximum resolution $(\AA)$ & 2.10 & 2.10 & 1.78 & 2.00 & 3.00 \\
\hline \multicolumn{6}{|l|}{ Data collection and processing } \\
\hline Space group & $I 222$ & $I 222$ & $I 222$ & $C 2$ & $I 222$ \\
\hline Resolution limits $(\AA)$ & $25-2.10$ & $25-2.10$ & $20-1.78$ & $44.49-2.00$ & $59.55-3.00$ \\
\hline Wavelength $(\AA)$ & 1.2852 & 1.2855 & 1.2825 & 1.6064 & 1.7266 \\
\hline No. of observed reflections & $124,019(15,613)$ & $129,536(18,012)$ & $205,787(16,293)$ & $109,104(16,014)$ & $25,156(3,637)$ \\
\hline No. of unique reflections & $21,295(3,071)$ & $216,57(3,094)$ & $34,120(4,707)$ & $31,103(4,417)$ & $6,444(918)$ \\
\hline Redundancy & 5.8 & 6.0 & 6.0 & 3.5 & 3.9 \\
\hline$R_{\text {pim }}{ }^{\mathrm{a}}$ & $0.057(0.210)$ & $0.065(0.247)$ & $0.069(0.490)$ & $0.058(0.327)$ & $0.078(0.246)$ \\
\hline Completeness $(\%)$ & $99.9(99.6)$ & $99.9(100.0)$ & $99.2(95.3)$ & $97.7(94.4)$ & $91.3(92.0)$ \\
\hline$\langle I / \sigma I\rangle$ & $15.4(5.2)$ & $14.7(2.9)$ & $10.2(1.6)$ & $9.0(2.1)$ & $15(5.1)$ \\
\hline \multicolumn{6}{|l|}{ MAD phasing statistics } \\
\hline No. of sites & 1 & 1 & & & \\
\hline Anomalous multiplicity & $2.9(2.5)$ & $3.0(2.9)$ & & & \\
\hline Anomalous completeness & $97.7(94.6)$ & $99.2(98.5)$ & & & \\
\hline FoM (centric/acentric) $2.96 \AA$ & $0.44(0.15)$ & & & & \\
\hline FoM DM $2.10 \AA$ & 0.91 & & & & \\
\hline Phasing power & 1.3 & 0.7 & & & \\
\hline \multicolumn{6}{|l|}{ Refinement statistics } \\
\hline Resolution $(\AA)$ & & & $23.91-1.80$ & $44.46-2.00$ & $46.25-3.00$ \\
\hline Reflections used & & & 31474 & 30458 & 6444 \\
\hline$R_{\text {work }}(\%)$ & & & 17.79 & 19.30 & 18.90 \\
\hline$R_{\text {free }}{ }^{\mathrm{b}}(\%)$ & & & 21.15 & 25.40 & 25.20 \\
\hline Mean protein $B$ factors $\left(\AA^{2}\right)$ & & & 14.00 & 15.77 & 16.06 \\
\hline No. of water molecules & & & 320 & 302 & 8 \\
\hline \multicolumn{6}{|c|}{ Ramachandran plot ${ }^{\mathrm{c}}$ : residues other than Gly and Pro in } \\
\hline Most favored regions (\%) & & & 99.54 & 95.24 & 91.71 \\
\hline Additional allowed regions $(\%)$ & & & 0.46 & 3.85 & 6.45 \\
\hline Disallowed regions (\%) & & & 0 & 0.91 & 1.84 \\
\hline PDB code & & & 2XB4 & $3 \mathrm{LOS}$ & $3 \mathrm{LOP}$ \\
\hline
\end{tabular}

Values in parentheses refer to the highest-resolution bin. $\mathrm{Zn}-\mathrm{AK} \mathrm{Zn}^{2+}$ form of adenylate kinase, $\mathrm{Co}-\mathrm{AK} \mathrm{Co}^{2+}$ form of adenylate kinase, $\mathrm{Fe}-\mathrm{AK}$ $\mathrm{Fe}^{2+}$ form of adenylate kinase

$A S U$ asymmetric unit, $M A D$ multiwavelength anomalous dispersion, FoM, DM, PDB Protein Data Bank

${ }^{\mathrm{a}} R_{\mathrm{pim}}=\sum_{h k l} \sqrt{\frac{1}{N-1}} \sum_{i}\left|I_{i}(h k l)-\overline{I(h k l)}\right| / \sum_{h k l} \sum_{i} I_{i}(h k l)$

${ }^{\mathrm{b}} R_{\text {free }}$ is calculated for a randomly chosen $5 \%$ of the reflections for each data set

${ }^{\mathrm{c}}$ Calculated using COOT validation 
molecules, and two molecules of tartaric acid from the crystallization solution. The initial TLS refinement was done by treating the whole protein as a single TLS entity. Later refinement cycles included ten separate TLS entities, identical for all structures. The final refinement statistics are presented in Table 1. One glycerol molecule was found in the asymmetric unit in both $\mathrm{Zn}-\mathrm{AK}$ and Fe-AK. The region 206-223 is partially disordered with no visible density for residue 209 . The residues with disordered side chains were stubbed at $\mathrm{C} \beta$, keeping the same label. Only eight water molecules could be located in the final Fe-AK structure.

\section{Results and discussion}

\section{Structure overview}

The crystal structures of $\mathrm{Zn}$-AK from native $\mathrm{AK}$ from D. gigas and recombinant $\mathrm{Co}-\mathrm{AK}$ and $\mathrm{Fe}-\mathrm{AK}$ were determined to $1.8-, 2.0-$, and $3.0-\AA$ resolution, respectively. A summary of data collection and refinement statistics is presented in Table 1. Zn-AK and Fe-AK crystallized as monomers in the asymmetric unit in space group I222, whereas Co-AK crystallized in space group $C 2$ as a dimer in the asymmetric unit. Superposition of the three structures yields root mean square deviation (RMSD) values of $0.10 \AA$ (Zn-AK vs. Co-AK) and $0.37 \AA$ (Zn-AK vs. Fe-AK), respectively, indicating that all three holo forms of AK have almost identical structures. The most striking observation is that replacement of the metal ion has no visible effect on the structure compared with the wild type.

Like the other AK structures, Zn-AK, Co-AK, and FeAK contain the characteristic LID (residues 125-163) and core (residues 1-124 and 164-223) domains, which also include the AMP binding region. The LID domain harbors the $\mathrm{Cys}^{129}-\mathrm{X}_{5}$ - $\mathrm{His}^{135}-\mathrm{X}_{15}$-Cys ${ }^{151}-\mathrm{X}_{2}$-Cys ${ }^{154}$ - motif, which is responsible for metal binding in a tetrahedral fashion. The core domain encloses the AMP binding region (residues 31-60) (Fig. 1). It is composed of a five-stranded $\beta$ sheet surrounded by seven $\alpha$-helices. The LID and the core domains are connected by two $\alpha$-helices (residues 116-123 and residues 165-173) which function as a hinge. $\mathrm{Zn}-\mathrm{AK}$ and Fe-AK have one molecule of glycerol and Co-AK has one molecule of tartaric acid bound in the core domain near residues $10-14$ in the substrate binding region. In the structures, glycerol and tartaric acid are found close to Arg126 of the connecting $\alpha$-helices or "hinge" and are strongly bound to the backbone nitrogen atom of Gly10 and Gly12, respectively (Fig. 1a). These interactions might help to stabilize this particular conformation of the enzyme. $B$-factor distributions of these structures show that the residues in the LID domain are in the same range as for the rest of the structure, showing that it is a well-defined region of the AK molecule.

To confirm the character of the metal ions, anomalous data were collected for both the $\mathrm{Zn}-\mathrm{AK}$ and Co-AK at three different wavelengths, corresponding to the zinc or the cobalt absorption peak, the zinc or the cobalt edge, and to a remote wavelength, respectively. Incorporation of $\mathrm{Co}^{2+}$ was verified with a fluorescence scan spanning both the zinc and the cobalt edges. A clear signal was found for cobalt, whereas no zinc was detected (Fig. S1). To further confirm that the incorporated metal was indeed cobalt, an anomalous map was calculated using multiwavelength anomalous dispersion data sets collected at the cobalt absorption peak and edge (1.6029 $\AA$ has an anomalous signal for cobalt $f^{\prime \prime} 4.0 \mathrm{e}^{-}$, whereas the signal for zinc is $0.7 e^{-}$at this wavelength). The next data set was collected at the cobalt edge with anomalous signal $f^{\prime \prime} 2.2 e^{-}$. The anomalous map was calculated using the data set collected at the cobalt absorption peak (Fig. 1). A strong anomalous peak corresponding to the cobalt atom in the metal binding site was found. This peak was noticeably weaker at the remote wavelength. For the confirmation of the iron in $\mathrm{Fe}-$ $\mathrm{AK}$, the same fluorescence scan procedure was done at the peak wavelength and an anomalous map was calculated only using the data set collected at the iron absorption peak (1.72 ̊).

\section{LID domain and metal coordination}

The AK family is divided into two types, short and long $\mathrm{AK}$, on the basis of the polypeptide chain length, with the longer variants evidencing a larger LID domain with an insertion of 20-30 residues [30]. The short AK conserve their catalytic activity despite the reduced LID domain (less than 11 residues), but seem to lose the high specificity for ATP [31]. The AK from D. gigas is of the long type, with a longer LID domain that consists of 28 residues (residues 125-163). The distance between the LID domain and the core domain is $12 \AA$ (distance between $\mathrm{C} \alpha$ of $\operatorname{Arg} 12$ and Gly126; Fig. 5). This distance is in accordance with other structurally characterized AK that are in the open state (e.g., AK from Aquifex aeolicus). This also confirms that the LID domain of the structures of AK from D. gigas is in the open state. Arg126 and Arg164 residues, which are located in the hinge region, take part in the LID domain and mediate catalysis because of their electrostatic interactions with phosphate groups of the substrates. When the substrate is present, these two Arg residues drag the LID domain closer to the core domain [4]. The conformation of the LID domain is more open in these structures compared with substrate-bound AK reported earlier. The crystal structures reported here show that the LID domain is stabilized by strong backbone hydrogen-bond 
Fig. 1 Structures of the three metal-bound forms of adenylate kinase (AK) from Desulfovibrio gigas. a Overall structure of the $\mathrm{Co}^{2+}$ form of AK (Co-AK) showing the LID domain (coral), core domain (violet), and AMP binding domain (green) and also the tartaric acid, bound close to the Walker motif (tan) and the Arg residue (Arg126) of the "hinge" (tan). b Superposition the structures of the $\mathrm{Zn}^{2+}$ form of $\mathrm{AK}$ ( $\left.\mathrm{Zn}-\mathrm{AK}\right)$, $\mathrm{Co}-\mathrm{AK}$, and the $\mathrm{Fe}^{2+}$ form of AK (Fe-AK). c-e The anomalous maps of the metal site in $\mathrm{Zn}-\mathrm{AK}$ (contoured at $3 \sigma$ ), Co-AK (contoured at $3.7 \sigma$ ), and Fe-AK (contoured at 2.8 $\sigma$ ), respectively. Pictures were prepared using CCP4mg [45]

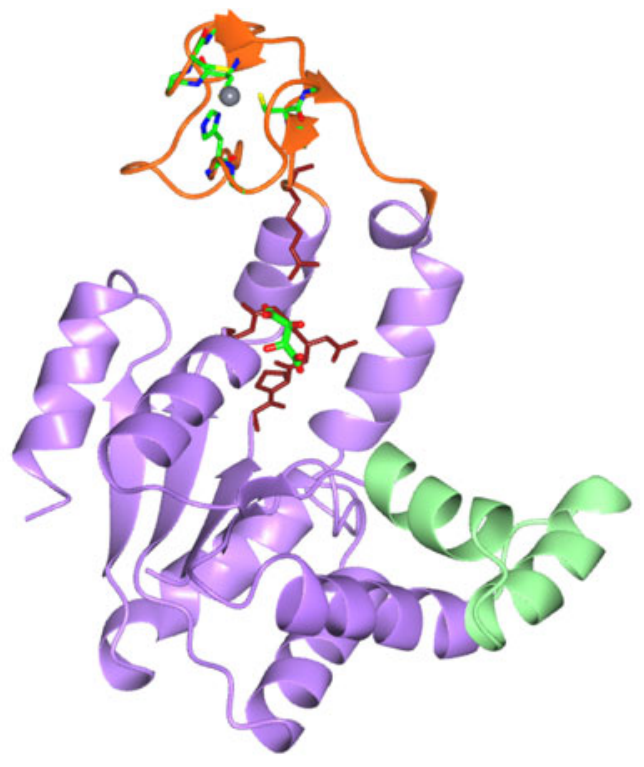

(A)

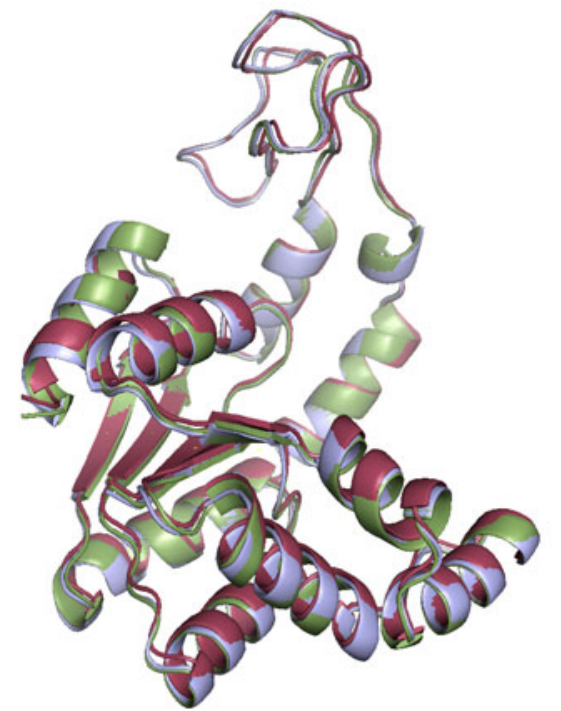

(B)

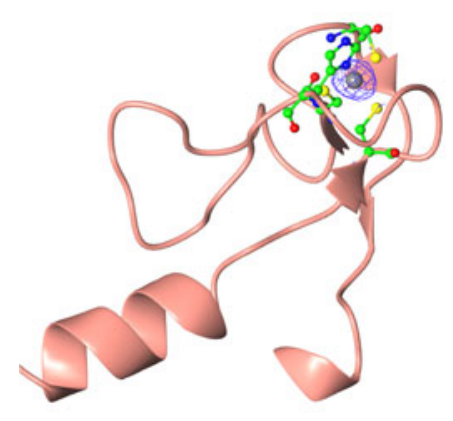

(C)

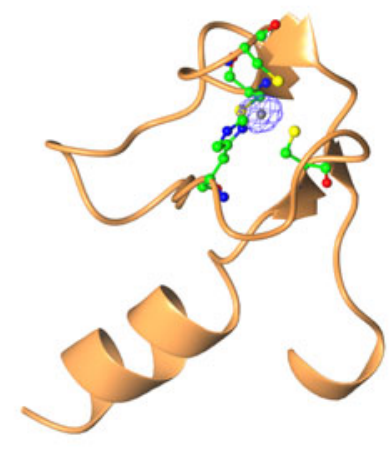

(D)

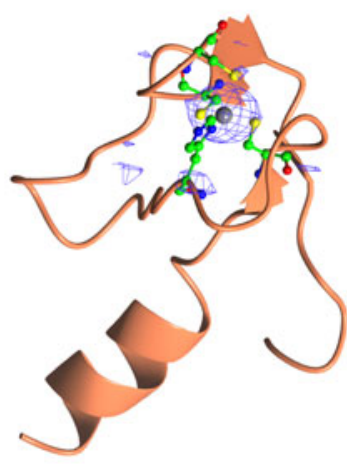

(E) interactions and few attractive hydrogen-bond interactions between the side chains. The residues involved in the hydrogen-bonding network are Asn127, Ile128, Arg137, Asn138, Ile144, and Ser159. Apart from these, several close interactions between the residues of the LID and the core domains and also with its symmetry mates contribute to the stabilization of the open conformation in the crystal. These strong interactions along with the metal chelation in the LID domain may enhance the thermal stability of the enzymes and also give it the structural integrity that makes it move as a rigid block towards the ATP binding pocket at the time of catalysis [14]. Binding of a metal ion ( $\mathrm{Zn}, \mathrm{Co}$, or $\mathrm{Fe}$ ) to the LID domain, as was shown previously, was found to provide a thermal stabilizing effect on the protein [18]. The $T_{\mathrm{m}}$ values for the apo and holo forms of $\mathrm{AK}$ from D. gigas at $\mathrm{pH} 10$ produced in vitro were 39.7 and $45.0^{\circ} \mathrm{C}$, respectively ( $T_{\mathrm{m}} \mathrm{Co}-\mathrm{AK} 46.1{ }^{\circ} \mathrm{C}$ and $\left.T_{\mathrm{m}} \mathrm{Zn}-\mathrm{AK} 44.3{ }^{\circ} \mathrm{C}\right)$.

The LID domain shares the same topology as a zinc finger domain present in other zinc-containing AK [4]. The metal ions $\left(\mathrm{Co}^{2+}, \mathrm{Zn}^{2+}\right.$ or $\left.\mathrm{Fe}^{2+}\right)$ are tetrahedrally coordinated to the 26-residue-long sequence $\mathrm{Cys}^{129}-\mathrm{X}_{5}$-His ${ }^{135}$ $\mathrm{X}_{15}$-Cys ${ }^{151}-\mathrm{X}_{2}$-Cys ${ }^{154}$-. The metal is located in an identical position in each structure. The distance between the ligating atoms and the metal ion is in the range 2.13-2.42 $\AA$ for $\mathrm{Zn}^{2+}, 2.00-2.42 \AA$ for $\mathrm{Co}^{2+}$, and $1.98-2.60 \AA$ for $\mathrm{Fe}^{2+}$, respectively. The overall topology and conformation of the LID domain remains unchanged in all the structures. This observation also supports the idea that the bound metal atoms in AK of Gram-negative bacteria are structural in nature, not catalytic. The substitution of zinc ion from the zinc finger motif by other redox metal atoms such as iron, cobalt and nickel has been reported, and it was shown that these metals exhibit lower affinity than zinc [32].

Core domain

The core domain is connected to the LID domain by residues 116-123 and 165-173. The latter are part of the 
counterweight loop [12], which is believed to control the movement of the LID domain during catalysis. This core domain mainly consists of a five-stranded $\beta$-sheet surrounded by five helices that keep the integrity of the tertiary structure of the enzyme. A Walker motif $[33,34]$ with conserved sequence G-X-X-G-X-G-K is present in the Nterminal region. This represents the phosphate binding loop characteristic of all AK. Glaser et al. [19, 35] have proposed that the sequence -G-F-P-R- (Gly84-Phe85-Pro86Arg87) present in the core domain plays a crucial role in stabilizing the tertiary structure of the enzyme. In all our three structures, Pro86 adopts a cis conformation similarly to the other structurally characterized AK. A major difference between our structures and the ones of substratebound AK is in the AMP binding region (residues 31-60). In the substrate-bound structures this region is rotated toward the core domain by as much as $5 \AA$.

Normal mode analysis of the LID domain

Normal mode analysis (NMA) was performed to address the influence of the nature of the metal ion in the dynamics of the LID and AMP binding domains [36].

The lowest frequency modes obtained (Fig. 2) exhibited good correlation with the experimentally observed changes in conformation [37-40]. The LID domain and the AMP binding region in the core domain are directly involved in the dynamic event during catalysis, closing over the enzyme's ATP and AMP binding sites. Computational pathway analysis of ligand-free E. coli $\mathrm{AK}[38,39]$ and the crystal structures of the conformational substates of $A$. aeolicus along this pathway toward the closed form have shown the changes in these two specified regions along the reaction pathway [41]. The most striking observation was that changing the metal did not influence the directionality of the movement of the LID domain. This further confirms that the role of the metal is to maintain the structure of the LID domain, allowing it to move as a rigid body.

Structural comparison with other AK

Several crystal structures of AK have been reported from other organisms [16-18]. A quick comparison of $\mathrm{Zn-AK}$ with members of each family of AK shows, as expected, that it shares more similarities with those that harbor metal atoms in the LID domain. The core domain is strikingly similar in all the structures. The topology of the LID domain and AMP/NMP binding regions shows most resemblance with the structures of enzymes that are devoid of any substrate. A search in the DALI server [42] revealed that the human AK isoform 2 (AK2; PDB code 2c9y, $z$ score of 22.8) was the closest structure available in the PDB. This AK also belongs to the long LID domain group.

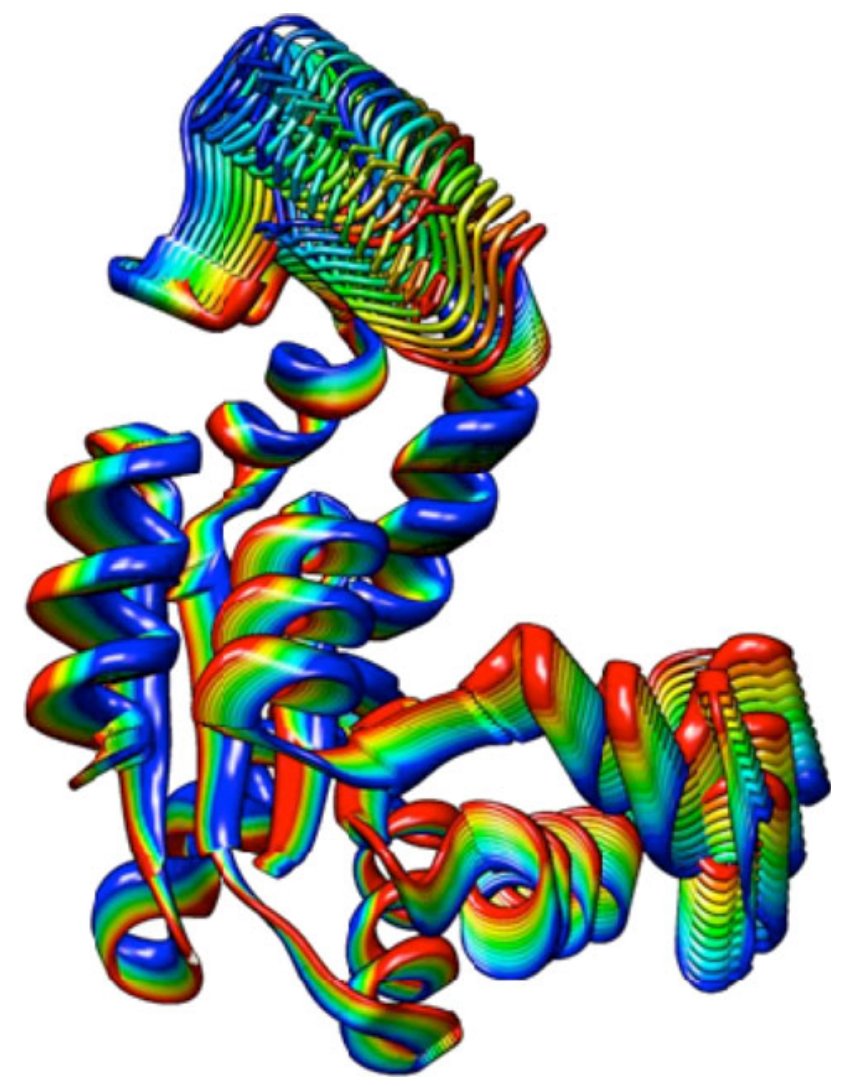

Fig. 2 Normal mode analysis (NMA) of DgAK. Lowest frequency mode of AK from D. gigas calculated using the EL NEMO server for NMA analysis [41] showing the dynamic fluctuation of the LID domain along the reaction pathway

Since all three structures studied share very similar overall structures, we will use only $\mathrm{Zn}-\mathrm{AK}$ in the following discussion. Zn-AK and human AK2 share 28\% sequence identity and their superposition yields an RMSD of $2 \AA$ (Fig. 3). The main difference between these two AK isoforms is the lack of a metal atom in the LID domain. The superposition of the core domains of these two proteins indicates similar secondary structures, but there is a significant change in the conformation of the LID domain (Fig. 3b). Since the human AK2 structure has a substrate bound, the LID domain is in the closed conformation. Instead of the three Cys and His residues that are present in Zn-AK, AK2 has Arg, His, Asp, and Tyr residues, which are not capable of metal-binding. These four residues are involved in a strong hydrogen-bond network that holds the LID domain together. Comparison of Zn-AK with the apo form of the AK from E. coli also shows similarities between the two structures. The core and AMP binding regions share similar secondary structure features. As expected, the LID domain of $\mathrm{Zn}-\mathrm{AK}$ is in a more open conformation owing to the strong crystal packing interactions and has a different topology owing to the presence of the metal ion. 


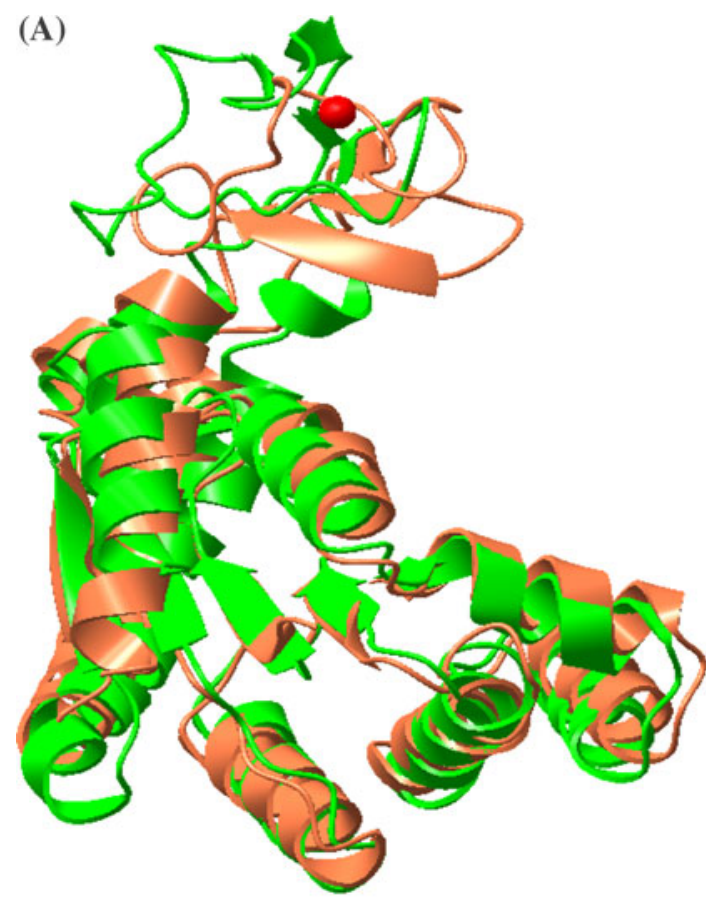

(B)

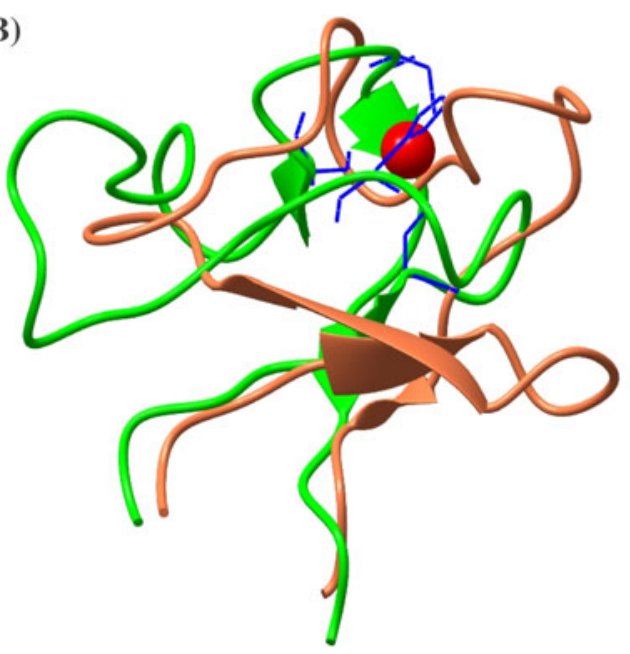

Fig. 3 Structure comparison of $\mathrm{DgAK}$ and human $\mathrm{AK}$ isoform 2 (AK2). a Superposition of Zn-AK (green) and human AK2 (coral). b Superposition of the LID domain of $\mathrm{Zn}-\mathrm{AK}$ and human AK2

One other structure of $\mathrm{AK}$ from Gram-negative bacteria-AK from A. aeolicus [38] - has been reported, as have several structures from Archaea-AK from Methanococcus voltae and Methanococcus thermolithotrophicus [43] (Gram negative) and AK from Methanococcus maripaludis [9, 43] and Sulpholobus acidocaldarius (variable response to the Gram stain) [10] — but none of them contain a metal atom in their LID domain. The multiple sequence alignment of AK from D. gigas and these three reported $\mathrm{AK}$ is presented in Fig. 4. Both AK from $M$. voltae and AK from $S$. acidocaldarius are distantly related to the other AK. They are trimeric in solution, and contain a central $\beta$-sheet and a short LID domain. Only a comparison study with the enzyme from A. aeolicus was performed. The structure of the apo form of AK from A. aeolicus showed three molecules in the asymmetric unit, each with a different conformation of the LID domain. Superposition of $\mathrm{Zn}-\mathrm{AK}$ and AK from A. aeolicus in the most open conformation yields an RMSD of $2.18 \AA$. These proteins share $34 \%$ sequence identity. Both of them contain a Walker motif, G-X-X-G$\mathrm{X}-\mathrm{G}-\mathrm{K}-$, present in the N-terminal region of the enzyme. A four-residue-long motif -G-F-P-R- is present in their core domain. The Arg residues (Arg126 and Arg164) in the hinge are also present in both structures. The distance between the $\mathrm{C} \alpha$ atom of one of the Arg residues present in the hinge and the $\mathrm{C} \alpha$ atom of one of the Gly residues from the Walker motif of the core domain can be used as a measure of the extent of opening of the LID domain. The Arg residues of the hinge and the Walker motifs are conserved in almost all AK with a long LID domain (Fig. 4). The topology of the LID domain is also different in $\mathrm{Zn}-\mathrm{AK}$, where two reverse turns allow the zinc-binding residues to be placed at the correct positions. The AMP binding regions of the core domain are very similar. The LID domain in the substrate-bound structure of AK from $A$. aeolicus is in the closed conformation and the distance between the LID domain and the core domain is $10.6 \AA$, whereas in open conformation they are $15.4 \AA$ apart. The "hinge" Arg residues (Arg126 and Arg164) have shifted almost $5 \AA$ toward the substrate, accompanied by the LID domain. The AMP binding domain of AK from A. aeolicus (corresponding to residues $31-65$ in $\mathrm{Zn}-\mathrm{AK}$ ) is also in the closed conformation in the substrate-bound form, in contrast to $\mathrm{Zn}-\mathrm{AK}$.

We have also compared the structural features of Zn-AK with other zinc-containing AK from Grampositive bacteria. From the structures we have examined, we chose for the comparison study the AK from B. globisporus [44], B. subtilis, and B. stearothermophilus. Like the AK from D. gigas, these also have the LID domain and the AMP binding domain that close during catalysis. Their secondary structures are very similar in spite of the fact that the sequence identity is only approximately $30 \%$ and different zinc-binding sites are present. Like the AK from B. globisporus and B. subtilis, in $\mathrm{Zn}-\mathrm{AK}$ the zinc atom is also bound to three Cys and one other residue: in this case it is His135. All these enzymes share an almost identical secondary structure in their core domain. All these enzymes were crystallized with AMP bound, whereas $\mathrm{Zn}-\mathrm{AK}$ is in the apo form. As expected, $\mathrm{Zn}$-AK has indeed the AMP binding domain in a fully open conformation as compared with the other three enzymes (Fig. 5). The regions equivalent to residues 31-65 in the other three AK are bound to the AMP and come closer to the core domain, adopting a closed 
Fig. 4 Sequence comparison of AK with known structures. Multiple sequence alignment of the AK from D. gigas, Aquifex aeolicus, Sulpholobus acidocaldarius, and Methanococcus voltae.

Residues that bind the metal ion are marked with triangles. The connectors at the top show the Walker motif at the N-terminus and the -G-F-P-R- region (residues 84-87) in the core domain. The picture was made with Aline [46]

\section{D. gigas \\ A. aeolicus \\ S. acidocalduris M. voltae \\ D. gigas \\ A. aeolicus \\ S. acidocalduris M. voltae}

\section{D. gigas \\ A. aeolicus \\ S. acidocalduris M. voltae}

\section{D. gigas A. aeolicus S. acidocalduris M. voltae}

\section{D. gigas A. aeolicus S. acidocalduris M. voltae}

\section{D. gigas A. aeolicus S. acidocalduris M. voltae}

\section{D. gigas \\ A. aeolicus S. acidocalduris} M. voltae

D. gigas
A. aeolicus
S. acidocalduris
M. voltae

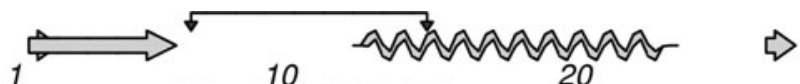

. MN I L I F GP NGS GKG T Q G N LVK D K Y S L A . M I L V F L GP PGA GKG T A K R L A K E K G F V $M K \ldots I G I V T G I \mathbf{P} G V G K S T V L A K V K E I \ldots$ $M K N K L V V V T G V P G V G G T T I T Q K A M E K \ldots$

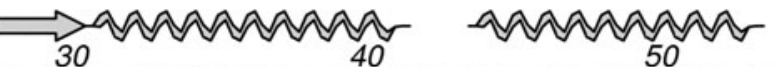
H I E S G GIF R E H I G G G T E L G K K A K E F I D R G D H I S T G D I L R E A V Q K G T P L G K K A K E Y M E R G E . L D N Q G I N N K I I N Y GD F M L T A L K . . L G Y . L S E E G I N Y K MVN F G TVME EVAQE... E N L

P D D I T I P M V LETLESKGKDGWL L D F P R

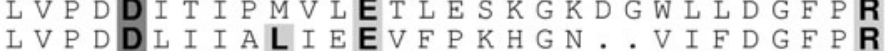
A K D R DEMRKL SVEKQK............ K

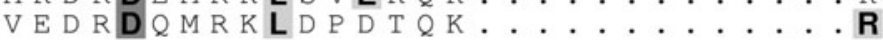

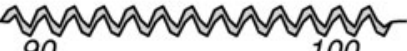

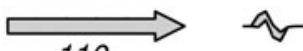
N T V Q A $Q K$ L E E A L Q E K GMK I N F V I ETL L P R E T VK Q A E A L D E M L E K K GLK V D H V L L E V P D E L $Q$ I D A A K G I A E A R A G E G Y L F I TH A V I R I $Q K$ L A G R K I A E MVK... E S P VVV D TH S T I K

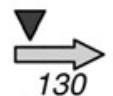

140

VAKN R I M GR R C K N N P H P N I F I E A I K P N VVIERLSGRRIN... P E T GE VYH V K Y N P P T P S G Y L P G L P S Y V I T E I N P S V I F L L E A D P K TP K G Y L G L P VWV L N E L N P D I I V V E T S G D

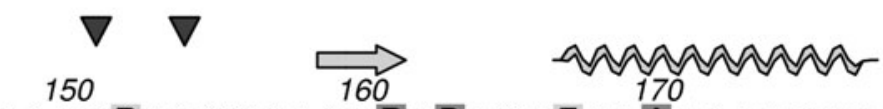
G D V C R V C G G A L S A R A D Q D E G A I N K R H D I Y 177 P P G V K V I . ....REDDKRE.V I K K R L E VY 165 I I L S R K K R T T R N R N D Y S D E S V I L E T I N F A 157 E I I I R R I D E T R N R. DLET T A IE E H I M N

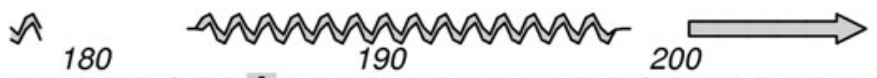
Y N T V D G T LA A A Y Y K N M A K E G F V Y I E L D G 207 R. . E Q T A P I I Y Y K K K I LR..... I I D A 187 R.... Y A A T A S A V L A G S T V K... V I V N V 178 R.... A A A M T Y G V L T G A T K .... I I Q N K 176

\section{0} Nana Nan

E G S I D S I K D T L L A Q L A . . . 223

SKPVEEVYRQVLEVIGD G N 206

E G D P S I A A N E I I R S M ... 194

N N L L D Y A V E E L I S V L R... 192 conformation. Owing to the absence of substrate, the LID domain in $\mathrm{Zn}-\mathrm{AK}$ is also more open than in these three structures. Nevertheless, the topology of the LID domain is very similar. All of these share the two reverse turns, a characteristic feature of the zinc-containing $\mathrm{AK}$ from Gram-negative bacteria [14], which facilitates the zinc coordination. The corresponding Arg residues (Arg124 and Arg166) in these three enzymes also show a 4 - $\AA$ shift in the substrate-bound form as compared with $\mathrm{Zn}-\mathrm{AK}$. As a consequence, the LID domain is shifted toward the closed conformation.

\section{Conclusion}

The structures of $\mathrm{Zn}-\mathrm{AK}, \mathrm{Co}-\mathrm{AK}, \mathrm{Fe}-\mathrm{AK}$ from the Gramnegative bacterium $D$. gigas have been determined. AK from $D$. gigas has the long type of the LID domain in a 


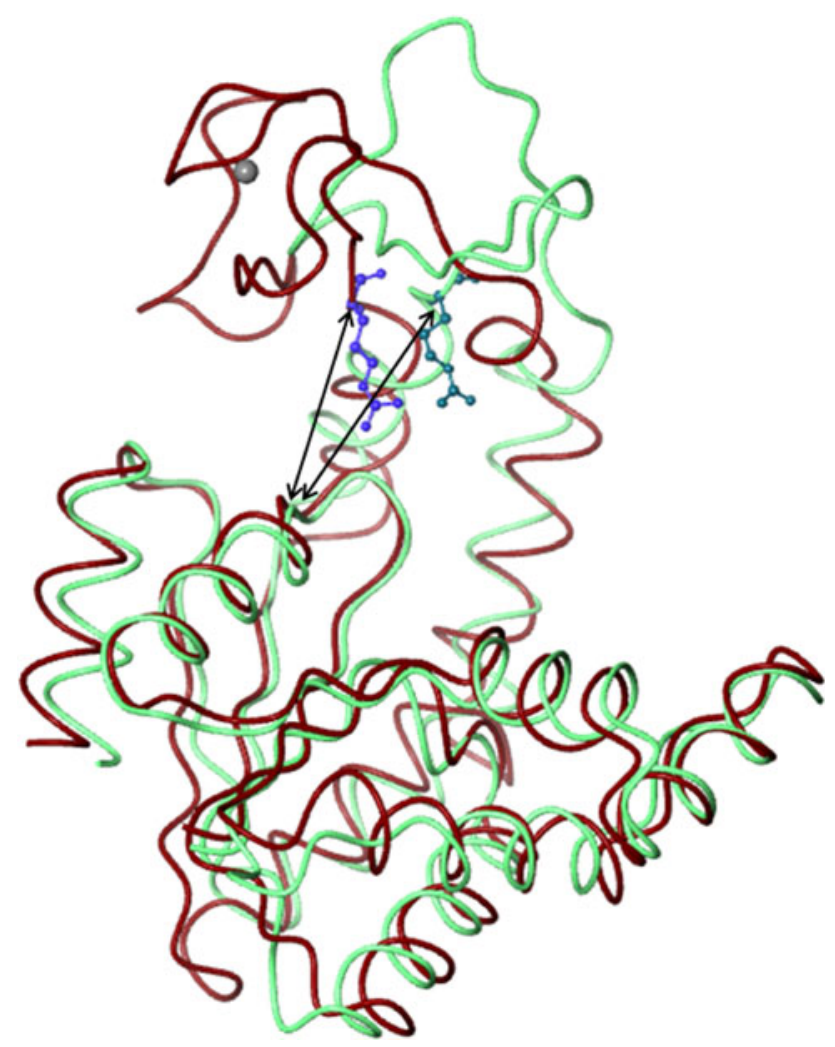

Fig. $5 \mathrm{DgAK}$ is in the open conformation. Superposition of Zn-AK (red) and the apo form of AK from A. aeolicus (light green). The distances between $\operatorname{Arg} 126$ (Arg124 for AK from A. aeolicus) and Gly12 for Zn-AK and AK from A. aeolicus are 12.35 and $15.37 \AA$, respectively

fully open conformation and contains the bivalent metal ion (zinc, cobalt, or iron) bound to the same metal-chelating motif (thee Cys residues and one His residue). The NMA study of AK from D. gigas shows the predicted directionality of the LID domain at the time of catalysis and its independence from the metal ion nature. Structural comparison of AK from D. gigas with other structurally similar AK from Gram-negative and Gram-positive bacteria revealed a similarity in the topology of the LID domain and also the conserved secondary structure of the core domain and confirmed the structural role of the metal ion.

Taken together, previous biochemical results and the structures presented herein further reinforce the notion that the metal ion is purely structural, contributing to the stability of the LID domain. The nature of the metal affects catalysis only indirectly, as it does not seem to influence the movement of the LID domain.

Acknowledgments This work was supported in part by the Fundação para a Ciência e a Tecnologia (FCT) project PPCDT/POCI/QUI/59119/ 2004 (Portugal), Acções Integradas Luso Espanholas E-62/06 (Portugal-Spain), and FCT Grants SFRH/BPD/20142/2006 (A.M.), SFRH/ BD/24744/2005 (A.V.K.), and SFRH/BPD/28380/2006 (O.Yu. G). We acknowledge the European Synchrotron Radiation Facility for provision of synchrotron radiation facilities.

\section{References}

1. Brune M, Schumann R, Wittinghofer F (1985) Nucleic Acids Res 13:7139-7151

2. Noda LH (1973) In: Boyer PD (ed) The enzymes, vol 8, part A. Academic Press, Orlando, pp 279-305

3. Müller CW, Schulz GE (1992) J Mol Biol 224:159-177

4. Berry MB, Phillips GN Jr (1998) Proteins Struct Funct Genet 32:276-288

5. Schulz GE (1992) Curr Opin Struct Biol 2:61-67

6. Yan H, Tsai MD (1999) Adv Enzymol Relat Areas Mol Biol 73:103-134

7. Bae E, Phillips GN Jr (2005) J Biol Chem 280:30943-30948

8. Vonrhein C, Bönisch H, Schäfer G, Schulz GE (1998) J Mol Biol 282:167-179

9. Davlieva M, Shamoo Y (2009) Proteins Struct Funct Genet 78:357-364

10. Perrier V, Burlacu-Miron S, Boussac A, Meier A, Gilles A (1998) Protein Eng 11:917-923

11. Wild K, Grafmüller R, Wagner E, Schulz GE (1997) Eur J Biochem 250:326-331

12. Gavel OY, Bursakov SA, Rocco GD, Trincão J, Pickering IJ, George GN, Calvete JJ, Shnyrov VL, Brondino CD, Pereira AS, Lampreia J, Tavares P, Moura JJG, Moura I (2008) J Inorg Biochem 102:1380-1395

13. Schlauderer GJ, Schulz GE (1996) Protein Sci 5:434-441

14. Perrier V, Burlacu-Miron S, Bourgeois S, Surewicz WK, Gilles A-M (1998) J Biol Chem 273:19097-19101

15. Müller CW, Schlauderer GJ, Reinstein J, Schulz GE (1996) Structure 4:147-156

16. Perrier V, Surewicz WK, Glaser P, Martineau L, Craescu CT, Fabian H, Mantsch HH, Barzu O, Gilles AM (1994) Biochemistry 33:9960-9967

17. Vielle C, Krishnamurthy H, Hyun H-H, Savchenko A, Yan H, Zeikus G (2003) Biochem J 372:577-585

18. Gavel OY, Bursakov SA, Pina DG, Zhadan GG, Moura JJ, Moura I, Shnyrov VL (2004) Biophys Chem 110:83-92

19. Glaser P, Presecan E, Delepierre M, Surewicz WK, Mantsch HH, Barzu O, Gilles A (1992) Biochemistry 31:3038-3043

20. Miura K, Inouye S, Sakai K, Takaoka H, Kishi F, Tabuchi M, Tanaka T, Matsumoto H, Shirai M, Nakazawa T, Nakazawa A (2001) J Biol Chem 276:13490-13498

21. Kladova AV, Gavel OY, Zhadan GG, Roig MG, Shnyrov VL, Bursakov SA (2009) Int J Biol Macromol 45(5):524-531

22. Kladova AV, Gavel OY, Boer DR, Mukhopaadhyay A, Texeira S, Shnyrov V, Moura I, Moura JJ, Romão MJ, Trincão J, Bursakov SA (2009) Acta Crystallogr F Struct Biol Crystallogr Commun 65:926-929

23. Leslie AGW (1992) Joint CCP4 and ESF-EACBM newsletters on protein crystallography 26

24. Kabsch W (1978) Acta Crystallogr Sect A Cryst Phys Diffr Theor Gen Crystallogr 34:827-828

25. Collaborative Computational Project Number 4 (1994) Acta Crystallogr D Biol Crystallogr 50:760-763

26. LaFortelle ED, Bricogne G (1997) In: Sweet JRM, Carter CW (eds) Methods in enzymology. Academic Press, New York, pp 472-494

27. McCoy AJ, Grosse-Kunstleve RW, Adams PD, Winn MD, Storoni LC, Read RJ (2007) J Appl Crystallogr 40:658-674 
28. Murshudov GN, Vagin AA, Dodson EJ (1997) Acta Crystallogr D Biol Crystallogr 53:240-255

29. Emsley P, Cowtan K (2004) Acta Crystallogr D Biol Crystallogr 60:2126-2132

30. Bellinzoni M, Haouz A, Graña M, Munier-Lehmann H, Shepard W, Alzari PM (2006) Protein Sci 15:1489-1493

31. Miyoshi K, Egi Y, Shioda T, Kawasaki T (1990) J Biochem 108:267-270

32. Hanas JS, Larabee JL, Hocker JR (2005) Molecular biology intelligence unit. Landes Bioscience, Austin, TX, 39-46

33. Walker JE, Saraste M, Runswick MJ, Gay NJ (1982) EMBO J 1:945-951

34. Dreusicke D, Schulz GE (1986) FEBS Lett 208:301-304

35. Gilles AM, Saint GI, Monnot M, Fermandjian S, Michelson S, Barzu O (1986) Proc Natl Acad Sci USA 83:5798-5802

36. Suhre K, Sanejouand YH (2004) Acta Crystallogr D Biol Crystallogr 60:796-799
37. Bahar I, Rader AJ (2005) Curr Opin Struct Biol 15:586-592

38. Miyashita O, Onuchic JN, Wolynes PG (2003) Proc Natl Acad Sci USA 100:12570-12575

39. Maragakis P, Karplus M (2005) J Mol Biol 229:494-501

40. Lou H, Cukier RI (2006) J Phys Chem B 110:12796-12808

41. Henzler-Wildman KA, Thai V, Lei M, Ott M, Wolf-Watz M, Fenn T, Pozharski E, Wilson MA, Petsko GA, Karplus M, Hubner CG, Kern D (2007) Nature 450:838-844

42. Holm L, Park J (2000) Bioinformatics 16:566-567

43. Criswell AR, Bae E, Stec B, Konisky J, Philips GN Jr (2003) J Mol Biol 330:1087-1099

44. Bae E, Philips GN Jr (2004) J Biol Chem 279:28202-28208

45. Potterton L, McNicholas S, Krissinel E, Gruber J, Cowtan K, Emsley P, Murshudov GN, Cohen S, Perrakis A, Noble M (2004) Acta Crystallogr D Biol Crystallogr 60:2288-2294

46. Bond CS, Schüttelkopf AW (2009) Acta Crystallogr D Biol Crystallogr 65:510-512 